\title{
A FAST PU MODE DECISION ALGORITHM FOR H.264/AVC TO HEVC TRANSCODING
}

\author{
Jiunn-Tsair Fang ${ }^{1}$, Zong-Yi Chen ${ }^{2}$, Tsai-Ling Liao ${ }^{2}$, and Pao-Chi Chang ${ }^{2}$ \\ ${ }^{1}$ Department of Electronic Engineering, \\ Ming Chuan University, Taoyuan, Taiwan \\ fang@mail.mcu.edu.tw \\ ${ }^{2}$ Department of Communication Engineering, \\ National Central University, Jhongli, Taiwan \\ pcchang@ce.ncu.edu.tw
}

\begin{abstract}
H.264/AVC has been widely applied to various applications. However, a new video compression standard, High Efficient Video Coding (HEVC), had been finalized recently. In this work, a fast transcoder from H.264/AVC to HEVC is proposed. The proposed algorithm includes the fast prediction unit $(P U)$ decision and the fast motion estimation. With the strong relation between H.264/AVC and HEVC, the motion vectors (MVs), residuals, and modes from each coding block of H.264/AVC can be reused to predict the current encoding PU of HEVC. Furthermore, the $M V$ variance from $H .264 / A V C$ is calculated to decide the search range of $P U$ and also to reduce the prediction mode. Simulation results show that the proposed method can save up to $53 \%$ of the encoding time and maintains the rate-distortion $(R-D)$ performance for HEVC.
\end{abstract}

\section{KEYWORDS}

H.264/AVC, HEVC, PU, Fast Algorithm, Transcoding

\section{INTRODUCTION}

H.264/AVC is currently one of the popular video compression standards [1]. However, with increasingly demands on the high quality of video services, previous video compression standards are no longer satisfied. The new video compression standard, High Efficient Video Coding (HEVC) [2], had been finalized in April 2013. During the transfer to HEVC, transcoders become practical tools continuing to serve users. A fast transcoder from H.264/AVC to HEVC focusing on the prediction unit $(\mathrm{PU})$ is proposed.

Video transcoding refers to converting the video content from one format into another. For the transcoder of H.264/AVC to HEVC, the video bit-stream of H.264/AVC is fully decoded, and some parameters are extracted. These parameters are mainly motion vectors (MVs), residuals, and modes from each coding block. With strong relation between these two standards, these extracted parameters can be reused for HEVC, and the HEVC encoder does not need to create the related parameters for the prediction. Figure 1 shows the typical fast transcoder architecture. The proposed method is to build up the relation from these extracted parameters of H.264/AVC to predict the PU of HEVC. Then, the original procedure to predict PU by HEVC encoder can be skipped, and therefore the encoding time of HEVC is reduced.

David C. Wyld et al. (Eds) : CCSIT, SIPP, AISC, PDCTA, NLP - 2014

pp. 215-225, 2014. (C) CS \& IT-CSCP 2014

DOI : $10.5121 /$ csit.2014.4218 
Most related works for the transcoding focus on the fast mode decision and fast motion estimation because the time saving is significant. For the fast mode decision, the relationship between the input block and the current block was explored in [3]. Jing et al. determined the optimal coding mode for the re-encoding process [4]. Zhang et al. determined the best PU by the proposed power spectrum model [5]. As for the fast motion estimation, MV refinement was proposed to have a better MV predictor [3]. Garrido-Cantos et al. proposed to reduce the motion search range for H.264/SVC with the information of H.264/AVC [6]. In [7], the authors proposed a fast mode decision algorithm for HEVC to H.264/AVC intra frame transcoding.

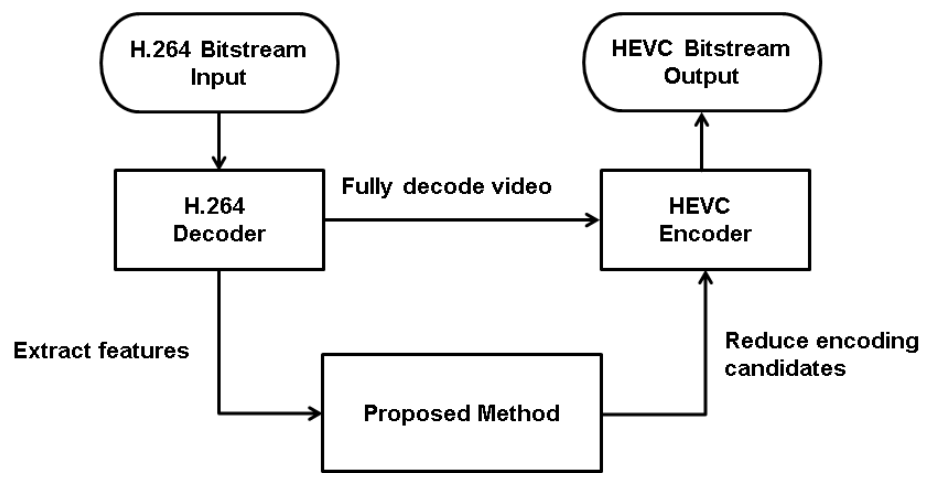

Figure 1. Typical fast transcoder architecture

Although promising results have been accomplished, their transcoders are applied for the same coding size between two standards. The coding unit (CU) sizes in depths 0 and 1 of HEVC are larger than the macroblock (MB) size of H.264/AVC. Thus, to design the transcoding from H.264/AVC to HEVC, various procedures are required for the different coding size prediction. A fast mode decision algorithm was proposed to explore the relationship between the input block and the current block for each depth of CU recently [8]. However, the PU was not considered in their method. Thus, a fast transcoding from H.264/AVC to HEVC focusing on PU is proposed.

The proposed algorithm consists of the fast mode decision and the fast motion estimation. Because of various coding sizes between H.264/AVC and HEVC, depths 0 and 1 and depths 2 and 3 of $\mathrm{CU}$ are applied various methods. The MVs, residuals, and modes information from H.264/AVC are reused for the PU encoder. As for the fast motion estimation, the MV variance from H.264/AVC is calculated to decide the search range of PU and also to reduce the prediction mode.

The rest of this paper is organized as follows. In Section 2, the proposed transcoding method is described. Section 3 shows the simulation results, and the conclusion is in Section 4.

\section{PROPOSED METHOD}

The proposed algorithm consists of the fast mode decision and the fast motion estimation. Before discussing the proposed algorithm, the preprocessing of the MV normalization is described.

\subsection{Motion Vector Normalization}

The MV extracted from various temporal references or various spatial sizes of H.264/AVC must be normalized to be the parameter predictor for HEVC. H.264/AVC applies multiple frames for the prediction, and the extracted MV may come from various temporal references. The MV normalization, denoted as $m v_{\text {norm }}$, is derived using (1): 


$$
m v_{n o r m}=\frac{1}{\alpha} m v_{n-\alpha},
$$

where $n$ is the current frame and $n-\alpha$ is the reference frame. Equation (1) shows that the MV normalization is to divide the frame number between the reference frame and the current frame. In other words, the extracted MV from the reference frame has a larger weighting if the reference frame is closer to the current frame.

H.264/AVC applies variable block sizes for the prediction, and the extracted MV may come from various sizes of the block. The MV normalization is to compare the area of the reference block with the area of the $4 \times 4$ block, which is derived using (2):

$$
m v_{\text {norm }}=\frac{\text { area of the reference block }}{\text { area of the } 4 \times 4 \text { block }} m v \text {. }
$$

Equation (2) shows that the extracted MV from the reference block has a larger weighting if the reference block has a larger area.

\subsection{Fast Mode Decision Algorithm}

In the proposed transcoder, the MVs, residuals, and modes information from H.264/AVC are reused to predict the PU of HEVC. However, the CU sizes in depths 0 and 1 of HEVC are larger than the MB size in H.264/AVC. Thus, the proposed fast mode algorithms for the transcoder to predict PU in depths 0 and 1 and PU in depths 2 and 3 are different.

\subsubsection{Fast Mode Decision Algorithm for Depth 0 and 1}

The proposed method for the transcoder to predict PU in depths 0 and 1 is described as follows. For the $2 \mathrm{~N} \times 2 \mathrm{~N}$ mode of PU, if all MVs in H.264/AVC are the same, the corresponding blocks in $\mathrm{HEVC}$ can be merged into a $2 \mathrm{~N} \times 2 \mathrm{~N}$ mode of $\mathrm{HEVC}$. For $2 \mathrm{~N} \times \mathrm{N}$ and $\mathrm{N} \times 2 \mathrm{~N}$ modes of $\mathrm{PU}$, the $\mathrm{MV}$ variance is defined as:

$$
V A R=\frac{1}{N} \sum_{n=1}^{N}\left(M V_{n}-\mu_{M V}\right)^{2},
$$

where $N$ is the total number of MVs in the reference block, and $\mu_{M V}$ is the average value of these MVs. According to (3), the calculations of the MV variances of the block $2 \mathrm{~N} \times \mathrm{N}$ and block $\mathrm{N} \times 2 \mathrm{~N}$ are as follows:

$$
\begin{aligned}
& \overline{V A R}_{2 N \times N}=\frac{1}{2}\left(V A R_{2 N \times N_{(U)}}+V A R_{2 N \times N_{(D)}}\right) \\
& \overline{V A R}_{N \times 2 N}=\frac{1}{2}\left(V A R_{N \times 2 N_{(L)}}+V A R_{N \times 2 N_{(R)}}\right),
\end{aligned}
$$

where $\overline{V A R}_{2 N \times N}$ is the average MV variance of upper and bottom blocks of the mode $2 \mathrm{~N} \times \mathrm{N}$, and $\overline{V A R}_{N \times 2 N}$ is the average $\mathrm{MV}$ variance of left and right blocks of the mode $\mathrm{N} \times 2 \mathrm{~N}$. The block segment is drawn in Figure 2. If the $\overline{V A R}_{X 2 N \times N} \geq 2 \times \overline{V A R}_{X N \times 2 N} \cap \overline{V A R}_{Y 2 N \times N} \geq 2 \times \overline{V A R}_{Y N \times 2 N}$, the mode $2 \mathrm{~N} \times \mathrm{N}$ in $\mathrm{PU}$ is skipped. On the other hand, if $\overline{V A R}_{X N \times 2 N} \geq 2 \times \overline{V A R}_{X 2 N \times N} \cap \overline{V A R}_{Y N \times 2 N} \geq 2 \times \overline{V A R}_{Y 2 N \times N}$, the mode $\mathrm{N} \times 2 \mathrm{~N}$ in PU is skipped. This is because a reference block with a larger MV variance is with less chance to be the candidate for the prediction. 

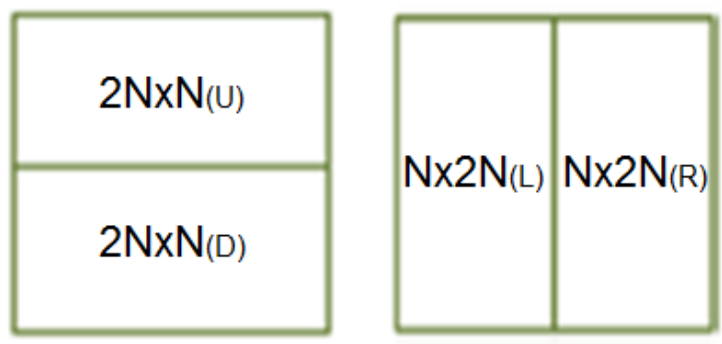

Figure 2. The block segment of the blocks $2 \mathrm{~N} \times \mathrm{N}$ and $\mathrm{N} \times 2 \mathrm{~N}$

For the asymmetric motion partition (AMP) mode in HEVC, the residual of each MB in H.264/AVC is applied to determine the candidate of the prediction. The residual is denoted as the sum of all the coefficients in the MB, derived using (4):

$$
\text { residual }=\sum_{i} \sum_{j}\left|\operatorname{coeff}\left(x_{i}, y_{j}\right)\right|
$$

where $\left(x_{i}, y_{j}\right)$ is the coefficient position of the $x$-axis and $y$-axis in an $\mathrm{MB}$, respectively. If the residual of $2 \mathrm{~N} \times \mathrm{N}_{(\mathrm{U})}$ is greater than the residual of $2 \mathrm{~N} \times \mathrm{N}_{(\mathrm{D})}$, the $2 \mathrm{~N} \times \mathrm{nD}$ mode is skipped. Otherwise, the $2 \mathrm{~N} \times \mathrm{nU}$ mode is skipped. As the residual of the $\mathrm{N} \times 2 \mathrm{~N}_{(\mathrm{L})}$ is greater than the residual of $\mathrm{N} \times 2 \mathrm{~N}_{(\mathrm{R})}$, the $\mathrm{nR} \times 2 \mathrm{~N}$ mode is skipped. Otherwise, $\mathrm{nL} \times 2 \mathrm{~N}$ mode is skipped. This is because a reference block with a larger residual implies that the block is with more content details and has a worse prediction, and the chance to adopt a mode with a worse prediction in H.264/AVC to PU is relatively low.

For the intra mode, it is selected only if the inter prediction has a poor prediction. Thus, if a $2 \mathrm{~N} \times 2 \mathrm{~N}$ block contains no any intra mode in H.264/AVC, the intra mode in HEVC is skipped.

\subsubsection{Fast Mode Decision Algorithm for Depth 2 and 3}

As for the depths 2 and 3, the MB size in H.264/AVC is the same as the CU size in HEVC. The proposed transcoder for fast algorithms in depths 2 and 3 are described as follows, respectively.

- $\quad$ Depth 2 fast mode decision algorithm:

$>$ If $\mathrm{H} .264 / \mathrm{AVC}$ is skip $=>$ just do $2 \mathrm{~N} \times 2 \mathrm{~N}$

$>$ If $\mathrm{H} .264 / \mathrm{AVC}$ is $16 \times 16=>$ do $2 \mathrm{~N} \times 2 \mathrm{~N}, 2 \mathrm{~N} \times \mathrm{N}$ and $\mathrm{N} \times 2 \mathrm{~N}$

$\rightarrow$ If residual $=0=>$ just do $2 \mathrm{~N} \times 2 \mathrm{~N}$

If $\mathrm{H} .264 / \mathrm{AVC}$ is $16 \times 8 \Rightarrow>$ do $2 \mathrm{~N} \times 2 \mathrm{~N}, 2 \mathrm{~N} \times \mathrm{N}, 2 \mathrm{~N} \times \mathrm{nU}$ and $2 \mathrm{~N} \times \mathrm{nD}$

$\rightarrow$ If residual $=0=>$ just do $2 \mathrm{~N} \times 2 \mathrm{~N}$ and $2 \mathrm{~N} \times \mathrm{N}$

$>$ If $\mathrm{H} .264 / \mathrm{AVC}$ is $8 \times 16 \Rightarrow$ do $2 \mathrm{~N} \times 2 \mathrm{~N}, \mathrm{~N} \times 2 \mathrm{~N}, \mathrm{~nL} \times 2 \mathrm{~N}$ and $\mathrm{nR} \times 2 \mathrm{~N}$

$\rightarrow$ If residual $=0=>$ just do $2 \mathrm{~N} \times 2 \mathrm{~N}$ and $\mathrm{N} \times 2 \mathrm{~N}$

$>$ If H.264/AVC is subMB $=>$ skip intra mode

$>$ If $\mathrm{H} .264 / \mathrm{AVC}$ is intra16 $=>$ do $2 \mathrm{~N} \times 2 \mathrm{~N}$ and intra

$>$ If $\mathrm{H} .264 / \mathrm{AVC}$ is intra $4=>$ do $2 \mathrm{~N} \times 2 \mathrm{~N}, 2 \mathrm{~N} \times \mathrm{N}, \mathrm{N} \times 2 \mathrm{~N}$ and intra mode

- $\quad$ Depth 3 fast mode decision algorithm:

If H.264/AVC is skip or $16 \times 16=>$ just do $2 \mathrm{~N} \times 2 \mathrm{~N}$

$>$ If H.264/AVC is $16 \times 8,8 \times 16$ or $8 \times 8=>$ skip intra

$\rightarrow$ If residual $=0=>$ just do $2 \mathrm{~N} \times 2 \mathrm{~N}$

If $\mathrm{H} .264 / \mathrm{AVC}$ is $8 \times 4=>$ skip intra $\mathrm{N} \times \mathrm{N}$

$\rightarrow$ If residual $=0=>$ skip $\mathrm{N} \times 2 \mathrm{~N}$ and intra mode 
If $\mathrm{H} .264 / \mathrm{AVC}$ is $4 \times 8 \Rightarrow>$ skip intra $\mathrm{N} \times \mathrm{N}$

$\rightarrow$ If residual $=0=>$ skip $2 \mathrm{~N} \times \mathrm{N}$ and intra mode

If $\mathrm{H} .264 / \mathrm{AVC}$ is $4 \times 4=>$ skip intra $\mathrm{N} \times \mathrm{N}$

$\rightarrow$ If residual $=0=>$ skip intra mode

If $\mathrm{H} .264 / \mathrm{AVC}$ is intra $16 \Rightarrow>$ do $2 \mathrm{~N} \times 2 \mathrm{~N}$ and intra $2 \mathrm{~N} \times 2 \mathrm{~N}$

$\rightarrow$ If residual $=0=>$ just do $2 \mathrm{~N} \times 2 \mathrm{~N}$

If $\mathrm{H} .264 / \mathrm{AVC}$ is intra4 $=>$ do $2 \mathrm{~N} \times 2 \mathrm{~N}$ and intra

\subsection{Fast Motion Estimation Algorithm}

In general, the procedure of the motion estimation spends the most encoding time. Two methods are applied for the proposed transcoder to reduce the search range of the motion estimation. One is to apply the adaptive motion search, and the other is to adjust the search range by the transition probability of the current encoding PU.

An adaptive motion search is proposed to reduce the search range. The reference search range (refSR) for each PU is defined using (5):

$$
\text { refSR }=\max _{i}\left(\max \left(\left|\mathrm{MV}_{\mathrm{X}_{i}}-\mathrm{AMVP}_{\mathrm{X}} \mathrm{I}, \mathrm{MVV}_{\mathrm{Y}_{i}}-\mathrm{AMVP}_{\mathrm{Y}}\right|\right)\right) .
$$

Equation (5) shows that to predict each PU, the search range is replaced by the refSR which is the maximum difference between the advance motion vector prediction (AMVP) of HEVC and each MV from H.264/AVC. Comparing with the fixed search range by HEVC, the proposed refSR is more flexible and effective. This is because the refSR adopts both information of MVs from H.264/AVC and HEVC. If the refSR is small, the MV prediction is with high accuracy. So, the search range can be small. On the other hand, if the refSR is large, the prediction is with low accuracy. The maximum difference between the predicted MVs as the search range shown in (5) can cover the worst condition. The difference between the H.264/AVC MV and the HEVC AMVP with respect to the $x$ and $y$ direction is drawn Figure 3 .
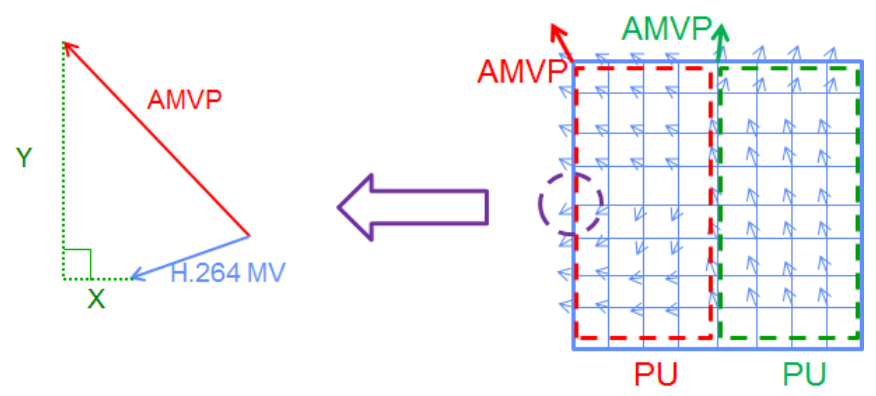

Figure 3. The difference between H.264/AVC MV and HEVC AMVP

In addition to the refSR, the mode transition probability from H.264/AVC to HEVC is also considered. If a PU in HEVC is with high probability to occur, the search range can be longer. Otherwise, the search range can be shorter if this PU rarely occurs.

For depths 2 and 3, the transition probability is directly measured from the mode in H.264/AVC to the PU in HEVC because the MB size in H.264/AVC is the same as the CU size in HEVC. Tables 1 and 2 show the transition probability of depths 2 and 3, respectively. The left column in Table 1 or 2 lists each mode in H.264/AVC and the above row lists the final PU by the HEVC 
encoder. For each row, it shows the transition probability of a mode in H.264/AVC to a PU in HEVC. The sum of the transition probability of each row is 1 .

Table 1. The transition probability for depth 2 .

HEVC

\begin{tabular}{|l|l|l|l|l|l|l|l|l|l|}
\hline & $16 \times 16$ & $16 \times 8$ & $8 \times 16$ & $16 \times 4$ & $16 \times 12$ & $4 \times 16$ & $12 \times 16$ & intra16 \\
\hline skip & 0.9772 & 0.0064 & 0.0082 & 0.0007 & 0.0013 & 0.0023 & 0.0012 & 0.0026 \\
\hline $16 \times 16$ & 0.8264 & 0.0395 & 0.0464 & 0.0189 & 0.0158 & 0.0222 & 0.0183 & 0.0108 \\
\hline $16 \times 8$ & 0.5681 & 0.2551 & 0.0514 & 0.0393 & 0.0315 & 0.0196 & 0.0174 & 0.0177 \\
\cline { 2 - 10 } \\
\cline { 2 - 10 } & $8 \times 16$ & 0.5427 & 0.0425 & 0.2778 & 0.0173 & 0.0155 & 0.0447 & 0.0352 & 0.0243 \\
\cline { 2 - 10 } & $8 \times 8$ & 0.3983 & 0.1674 & 0.1820 & 0.0491 & 0.0457 & 0.0670 & 0.0613 & 0.0291 \\
\cline { 2 - 9 } & 0.3528 & 0.1674 & 0.1771 & 0.0819 & 0.0745 & 0.0622 & 0.0557 & 0.0283 \\
\hline & $4 \times 8$ & 0.3342 & 0.1568 & 0.1827 & 0.0468 & 0.0399 & 0.1066 & 0.0972 & 0.0358 \\
\hline $4 \times 4$ & 0.3109 & 0.1706 & 0.1819 & 0.0619 & 0.0597 & 0.0936 & 0.0892 & 0.0323 \\
\hline intra16 & 0.6367 & 0.0073 & 0.0108 & 0.0032 & 0.0010 & 0.0014 & 0.0027 & 0.3369 \\
\hline intra4 & 0.2657 & 0.0414 & 0.0443 & 0.0054 & 0.0091 & 0.0237 & 0.0269 & 0.5835 \\
\hline
\end{tabular}

Table 2. The transition probability for depth 3 .

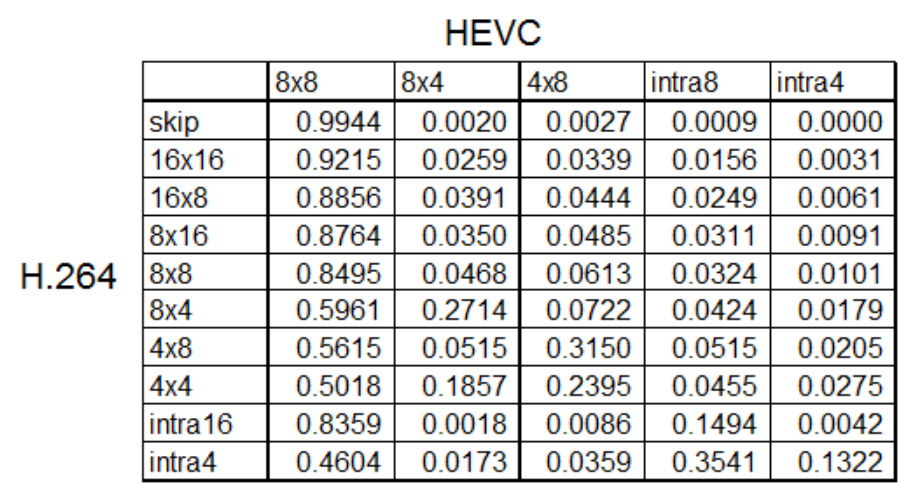

For depths 0 and 1 of CU, the CU size in HEVC is large than the MB size in H.264/AVC. If a block has been encoded as a subMB by H.264/AVC, the chance for this block to be merged into a larger size of $\mathrm{CU}$, encoded by HEVC, in depths 0 or 1 is low. In other words, if the current encoding CU contains more subMBs, already encoded by H.264/AVC, the chance for this CU to be encoded by HEVC in depths 0 and 1 is lower. The subMB denotes as a block with an $8 \times 8$ size or less. Thus, the transition probability can be obtained by counting the number of subMB within a CU. Counting the subMB is based on each $8 \times 8$ block, where an $8 \times 8$ block or all the smaller blocks within an $8 \times 8$ block are counted by 1 .

Tables 3 and 4 show the transition probability for depths 0 and 1, respectively. The left column in Table 3 or 4 lists the number of subMB for the current encoding CU, and the above row lists the final PU by the HEVC encoder. For each row, it shows the transition probability of the PU. The sum of the transition probability of each row is 1 . 
Table 3. The transition probability for depth 0 .

HEVC PU mode

\begin{tabular}{|c|c|c|c|c|c|c|c|c|c|}
\hline \multirow{6}{*}{ SubMB } & & $64 \times 64$ & $64 \times 32$ & $32 \times 64$ & $64 \times 16$ & $64 \times 48$ & $16 \times 64$ & $48 \times 64$ & intra64 \\
\hline & 0 & 0.6661 & 0.1002 & 0.1417 & 0.0172 & 0.0106 & 0.0163 & 0.0328 & \begin{tabular}{|l}
0.0151 \\
\end{tabular} \\
\hline & $1-4$ & 0.2508 & 0.2675 & 0.2930 & 0.0307 & 0.0233 & 0.0452 & 0.0786 & 0.0108 \\
\hline & $5-8$ & 0.1544 & 0.2897 & 0.3683 & 0.0337 & 0.0296 & 0.0500 & 0.0565 & 0.0179 \\
\hline & $9-12$ & 0.1379 & 0.3040 & 0.3894 & 0.0172 & 0.0259 & 0.0402 & 0.0439 & 0.0415 \\
\hline & $13-16$ & 0.0455 & 0.3482 & 0.4442 & 0.0417 & 0.0720 & 0.0152 & 0.0000 & 0.0333 \\
\hline
\end{tabular}

Table 4. The transition probability for depth 1 .

HEVC PU mode

\begin{tabular}{|c|c|c|c|c|c|c|c|c|c|}
\hline \multirow{6}{*}{ SubMB } & & $32 \times 32$ & $32 \times 16$ & $16 \times 32$ & $32 \times 8$ & $32 \times 24$ & $8 \times 32$ & $24 \times 32$ & intra32 \\
\hline & 0 & 0.6195 & 0.0979 & 0.1159 & 0.0343 & 0.0273 & 0.0440 & 0.0371 & 0.0240 \\
\hline & 1 & 0.3201 & 0.1640 & 0.2084 & 0.0619 & 0.0626 & 0.0836 & 0.0752 & 0.0242 \\
\hline & 2 & 0.2106 & 0.1787 & 0.2346 & 0.0765 & 0.0722 & 0.1037 & 0.1018 & 0.0219 \\
\hline & 3 & 0.1662 & 0.1845 & 0.2342 & 0.0938 & 0.0795 & 0.1152 & 0.1078 & 0.0188 \\
\hline & 4 & 0.1471 & 0.1859 & 0.2507 & 0.0840 & 0.0653 & 0.1250 & 0.1278 & 0.0141 \\
\hline
\end{tabular}

The search range of a PU is adjusted by a weighting factor which is assigned based on the PU transition probability. The assignment is listed in Table 5.

Table 5. The weighting table to the search range.

\begin{tabular}{|c|c|}
\hline Probability & Weighting, $\boldsymbol{W}$ \\
\hline $\mathrm{P} \leq 0.1$ & $\mathbf{0 . 6}$ \\
\hline $0.1<\mathrm{P} \leq 0.3$ & $\mathbf{0 . 8}$ \\
\hline $0.3<\mathrm{P} \leq 0.5$ & $\mathbf{1}$ \\
\hline $\mathrm{P}>0.5$ & $\mathbf{1 . 2}$ \\
\hline
\end{tabular}

Finally, the search range is defined using (6):

$$
\text { Search Range }=\min (W \times \text { refSR, original } S R) .
$$

The final search range is refSR multiplied by the weighting, but it cannot be greater than the default value, 64, of HEVC.

\section{EXPERIMENTAL RESULTS}

Simulations were taken by both JM, the software of H.264/AVC, version17.2 [9] with a QP value of 24, and HM, the software of HEVC, version 9.2 [10] with QP values 24, 27, 31, and 35. The input sequences were IPPP...P with different classes, and the test zone (TZ) fast search was applied in HEVC [10]. The test hardware was a PC with Intel I7-3770, 3.4GHz CPU, 16G RAM, and Windows 7 the professional system. Experimental results include the fast mode decision, fast motion estimation, and the overall performance. The overall performance is also compared with the performance proposed by [8]. 


\subsection{Results for the Fast Mode Decision Algorithm}

Table 6 lists the performance for the fast mode decision. The proposed method saves 32-35\% of the encoding time from the test sequences under four various QPs. In particular, the E class of sequences can save more encoding time because their contents are homogeneous, with smooth contents. Thus, larger size of blocks or more skip modes can be adopted by the proposed method.

Table 6. Experimental results for fast mode decision.

\begin{tabular}{|c|c|c|c|c|c|c|}
\hline & \multicolumn{3}{|c|}{$\mathrm{QP}=24$} & \multicolumn{3}{|c|}{$\mathrm{QP}=27$} \\
\hline Sequence & $\begin{array}{c}\triangle \mathrm{PSNR} \\
(\mathrm{dB})\end{array}$ & $\begin{array}{c}\triangle \text { Bitrate } \\
(\%)\end{array}$ & $\begin{array}{c}\triangle \text { EncTime } \\
(\%)\end{array}$ & $\begin{array}{c}\triangle \mathrm{PSNR} \\
(\mathrm{dB})\end{array}$ & $\begin{array}{c}\triangle \text { Bitrate } \\
(\%)\end{array}$ & $\begin{array}{c}\triangle \text { EncTime } \\
(\%)\end{array}$ \\
\hline ClassC_BasketballDrill & -0.041 & $1.64 \%$ & $-35.71 \%$ & -0.047 & $1.78 \%$ & $-34.57 \%$ \\
\hline ClassC_RaceHorses & -0.020 & $1.26 \%$ & $-27.35 \%$ & -0.030 & $1.49 \%$ & $-25.30 \%$ \\
\hline ClassD_BasketballPass & -0.026 & $1.28 \%$ & $-29.14 \%$ & -0.035 & $1.26 \%$ & $-28.38 \%$ \\
\hline ClassD_BlowingBubbles & -0.019 & $0.44 \%$ & $-27.14 \%$ & -0.018 & $0.52 \%$ & $-26.40 \%$ \\
\hline ClassE_vidyo1 & -0.042 & $0.41 \%$ & $-49.00 \%$ & -0.034 & $0.90 \%$ & $-48.79 \%$ \\
\hline ClassE_vidyo4 & -0.033 & $0.73 \%$ & $-43.89 \%$ & -0.040 & $0.71 \%$ & $-43.18 \%$ \\
\hline \multirow[t]{2}{*}{\begin{tabular}{|l|} 
Averge \\
\end{tabular}} & -0.030 & $0.96 \%$ & $-35.37 \%$ & -0.034 & $1.11 \%$ & $-34.44 \%$ \\
\hline & \multicolumn{3}{|c|}{$\mathrm{QP}=31$} & \multicolumn{3}{|c|}{$\mathrm{QP}=35$} \\
\hline Sequence & $\begin{array}{c}\triangle \mathrm{PSNR} \\
(\mathrm{dB})\end{array}$ & $\begin{array}{c}\triangle \text { Bitrate } \\
(\%)\end{array}$ & $\begin{array}{c}\triangle \text { EncTime } \\
(\%)\end{array}$ & $\begin{array}{c}\triangle \mathrm{PSNR} \\
(\mathrm{dB})\end{array}$ & $\begin{array}{c}\triangle \text { Bitrate } \\
(\%)\end{array}$ & $\begin{array}{c}\triangle \text { EncTime } \\
(\%)\end{array}$ \\
\hline ClassC_BasketballDrill & -0.050 & $1.91 \%$ & $-33.27 \%$ & -0.070 & $1.62 \%$ & $-32.50 \%$ \\
\hline ClassC_RaceHorses & -0.042 & $1.72 \%$ & $-23.61 \%$ & -0.043 & $1.73 \%$ & $-21.39 \%$ \\
\hline ClassD_BasketballPass & -0.047 & $1.55 \%$ & $-27.79 \%$ & -0.087 & $1.91 \%$ & $-27.18 \%$ \\
\hline ClassD_BlowingBubbles & -0.033 & $0.62 \%$ & $-24.74 \%$ & -0.033 & $0.88 \%$ & $-23.27 \%$ \\
\hline ClassE_vidyo1 & -0.038 & $0.60 \%$ & $-48.98 \%$ & -0.031 & $0.74 \%$ & $-49.41 \%$ \\
\hline ClassE_vidyo4 & -0.049 & $0.74 \%$ & $-43.18 \%$ & -0.046 & $1.03 \%$ & $-43.35 \%$ \\
\hline Averge & -0.043 & $1.19 \%$ & $-33.60 \%$ & -0.052 & $1.32 \%$ & $-32.85 \%$ \\
\hline
\end{tabular}

\subsection{Results for the Fast Motion Estimation Algorithm}

Table 7 lists the performance of the fast motion estimation. The proposed method saves approximately to $15 \%$ of the encoding time and maintains the R-D performance from the test sequences. In particular, the test sequence RaceHorses in class $C$ has better performance than other sequences. This is because this sequence is a high motion sequence, and the proposed adaptive search range can be more effective than the method of fixed search range. On the other hand, the test sequence Vidyo 1 in class $\mathrm{E}$ is a low motion sequence, the TZ search in HEVC can quickly find the best MV so that the time saving by the proposed method is reduced. 
Table 7. Experimental results for fast motion estimation.

\begin{tabular}{|c|c|c|c|c|c|c|}
\hline & \multicolumn{3}{|c|}{$\mathrm{QP}=24$} & \multicolumn{3}{|c|}{$\mathrm{QP}=27$} \\
\hline Sequence & $\begin{array}{c}\triangle \mathrm{PSNR} \\
(\mathrm{dB})\end{array}$ & $\begin{array}{c}\triangle \text { Bitrate } \\
(\%)\end{array}$ & $\begin{array}{c}\triangle \text { EncTime } \\
(\%)\end{array}$ & $\begin{array}{c}\triangle \mathrm{PSNR} \\
(\mathrm{dB})\end{array}$ & $\begin{array}{c}\triangle \text { Bitrate } \\
(\%)\end{array}$ & $\begin{array}{c}\triangle \text { EncTime } \\
(\%)\end{array}$ \\
\hline ClassC_BasketballDrill & -0.007 & $0.25 \%$ & $-15.70 \%$ & -0.008 & $0.38 \%$ & $-16.94 \%$ \\
\hline ClassC_RaceHorses & -0.001 & $0.34 \%$ & $-22.39 \%$ & -0.006 & $0.46 \%$ & $-22.67 \%$ \\
\hline ClassD_BasketballPass & -0.018 & $0.41 \%$ & $-17.14 \%$ & -0.017 & $0.25 \%$ & $-16.98 \%$ \\
\hline ClassD_BlowingBubbles & -0.006 & $0.11 \%$ & $-12.34 \%$ & -0.005 & $-0.21 \%$ & $-13.37 \%$ \\
\hline ClassE_vidyo1 & -0.011 & $-0.05 \%$ & $-7.78 \%$ & -0.008 & $-0.11 \%$ & $-7.46 \%$ \\
\hline ClassE_vidyo4 & -0.008 & $-0.04 \%$ & $-15.73 \%$ & -0.012 & $0.09 \%$ & $-15.70 \%$ \\
\hline \multirow[t]{2}{*}{$\begin{array}{r}\text { Averge } \\
\end{array}$} & -0.009 & $0.17 \%$ & $-15.18 \%$ & -0.009 & $0.14 \%$ & $-15.52 \%$ \\
\hline & \multicolumn{3}{|c|}{$\mathrm{QP}=31$} & \multicolumn{3}{|c|}{$\mathrm{QP}=35$} \\
\hline Sequence & $\begin{array}{c}\triangle \mathrm{PSNR} \\
(\mathrm{dB})\end{array}$ & $\begin{array}{c}\triangle \text { Bitrate } \\
(\%)\end{array}$ & $\begin{array}{c}\triangle \text { EncTime } \\
(\%)\end{array}$ & $\begin{array}{c}\triangle \mathrm{PSNR} \\
(\mathrm{dB})\end{array}$ & $\begin{array}{c}\triangle \text { Bitrate } \\
(\%)\end{array}$ & $\begin{array}{c}\triangle \text { EncTime } \\
(\%)\end{array}$ \\
\hline ClassC_BasketballDrill & 0.002 & $0.47 \%$ & $-16.95 \%$ & -0.033 & $-0.16 \%$ & $-16.29 \%$ \\
\hline ClassC_RaceHorses & -0.025 & $0.42 \%$ & $-24.48 \%$ & -0.020 & $0.40 \%$ & $-23.87 \%$ \\
\hline ClassD_BasketballPass & -0.030 & $0.01 \%$ & $-16.12 \%$ & -0.042 & $0.33 \%$ & $-14.84 \%$ \\
\hline ClassD_BlowingBubbles & -0.005 & $0.31 \%$ & $-13.64 \%$ & -0.024 & $0.49 \%$ & $-13.47 \%$ \\
\hline ClassE_vidyo1 & -0.010 & $-0.28 \%$ & $-7.31 \%$ & 0.002 & $-0.38 \%$ & $-7.02 \%$ \\
\hline ClassE_vidyo4 & -0.004 & $-0.41 \%$ & $-14.66 \%$ & 0.001 & $0.15 \%$ & $-13.25 \%$ \\
\hline Averge & -0.012 & $0.09 \%$ & $-15.53 \%$ & -0.019 & $0.14 \%$ & $-14.79 \%$ \\
\hline
\end{tabular}

\subsection{Results for the Overall Performance}

Table 8 lists the overall performance. The proposed algorithm can save 43-45\% of the encoding time. Comparing with [8], the proposed method performs better in the encoding time but increases the bitrate a little. In particularly, as the QP is closer to the original QP by H.264/AVC, the proposed method saves much of the encoding time. Therefore, as these two transcoding standards are with the closer QP assignment, the proposed algorithm can be more effective.

\section{CONCLUSIONS}

In this work, a fast transcoder from H.264/AVC to HEVC focusing on the PU is proposed. The proposed method consists of the fast mode decision and the fast motion estimation. Various fast mode algorithms are applied to the transcoder for depths 0 and 1, and depths 2 and 3, respectively. As for the fast motion estimation, adaptive search range with the mode transition probability is proposed. Experimental results show the algorithms of the fast mode decision and the fast motion estimation can save $32-35 \%$ and $15 \%$ of the encoding time, respectively. The overall encoding time can be reduced up to $53 \%$. In other words, more than half of the encoding time can be saved under the acceptable R-D performance. 
Table 8. Experimental results for overall algorithm.

\begin{tabular}{|c|c|c|c|c|c|c|}
\hline & \multicolumn{3}{|c|}{ Reference [8] } & \multicolumn{3}{|c|}{ Proposed Method } \\
\hline Sequence & $\begin{array}{c}\triangle \mathrm{PSNR} \\
(\mathrm{dB})\end{array}$ & $\begin{array}{c}\triangle \text { Bitrate } \\
(\%)\end{array}$ & $\begin{array}{c}\triangle \text { EncTime } \\
(\%)\end{array}$ & $\begin{array}{c}\triangle \mathrm{PSNR} \\
(\mathrm{dB})\end{array}$ & $\begin{array}{c}\triangle \text { Bitrate } \\
(\%)\end{array}$ & $\begin{array}{c}\triangle \text { EncTime } \\
(\%)\end{array}$ \\
\hline ClassB_Cactus & -0.030 & $0.38 \%$ & $-35.67 \%$ & -0.028 & $0.83 \%$ & $-44.41 \%$ \\
\hline ClassB_ParkScene & -0.021 & $0.16 \%$ & $-32.02 \%$ & -0.032 & $0.65 \%$ & $-44.97 \%$ \\
\hline ClassC_BasketballDrill & -0.045 & $0.97 \%$ & $-36.75 \%$ & -0.046 & $1.89 \%$ & $-46.57 \%$ \\
\hline ClassC_RaceHorses & -0.019 & $0.94 \%$ & $-41.24 \%$ & -0.019 & $1.69 \%$ & $-43.83 \%$ \\
\hline ClassD_BasketballPass & -0.054 & $1.59 \%$ & $-39.02 \%$ & -0.037 & $1.53 \%$ & $-42.09 \%$ \\
\hline ClassD_BlowingBubbles & -0.063 & $1.63 \%$ & $-33.50 \%$ & -0.020 & $0.67 \%$ & $-36.41 \%$ \\
\hline ClassE_vidyo1 & -0.062 & $-0.39 \%$ & $-43.43 \%$ & -0.057 & $0.30 \%$ & $-53.27 \%$ \\
\hline ClassE_vidyo4 & -0.042 & $-0.07 \%$ & $-43.61 \%$ & -0.038 & $0.98 \%$ & $-53.73 \%$ \\
\hline QP24 Averge & -0.042 & $0.65 \%$ & $-38.16 \%$ & -0.035 & $1.07 \%$ & $-45.66 \%$ \\
\hline ClassB_Cactus & -0.015 & $0.44 \%$ & $-37.16 \%$ & -0.028 & $1.15 \%$ & $-43.64 \%$ \\
\hline ClassB_ParkScene & -0.022 & $0.20 \%$ & $-33.55 \%$ & -0.036 & $0.82 \%$ & $-44.02 \%$ \\
\hline ClassC_BasketballDrill & -0.028 & $0.97 \%$ & $-37.48 \%$ & -0.050 & $2.22 \%$ & $-45.90 \%$ \\
\hline ClassC_RaceHorses & -0.029 & $1.09 \%$ & $-41.94 \%$ & -0.035 & $2.20 \%$ & $-43.45 \%$ \\
\hline ClassD_BasketballPass & -0.050 & $1.60 \%$ & $-39.52 \%$ & -0.051 & $1.61 \%$ & $-41.52 \%$ \\
\hline ClassD_BlowingBubbles & -0.068 & $1.03 \%$ & $-34.17 \%$ & -0.027 & $0.74 \%$ & $-36.25 \%$ \\
\hline ClassE_vidyo1 & -0.023 & $0.23 \%$ & $-45.84 \%$ & -0.042 & $1.13 \%$ & $-53.28 \%$ \\
\hline ClassE_vidyo4 & -0.026 & $-0.02 \%$ & $-45.98 \%$ & -0.047 & $0.79 \%$ & $-53.53 \%$ \\
\hline QP27 Averge & -0.033 & $0.69 \%$ & $-39.46 \%$ & -0.040 & $1.33 \%$ & $-45.20 \%$ \\
\hline ClassB_Cactus & -0.016 & $0.87 \%$ & $-38.26 \%$ & -0.048 & $1.27 \%$ & $-42.88 \%$ \\
\hline ClassB_ParkS & -0.025 & $0.25 \%$ & $-34.98 \%$ & -0.042 & $0.89 \%$ & $-43.04 \%$ \\
\hline ClassC_BasketballDrill & -0.029 & $1.08 \%$ & $-38.67 \%$ & -0.054 & $2.35 \%$ & $-44.99 \%$ \\
\hline ClassC_RaceHorses & -0.043 & $1.18 \%$ & $-42.41 \%$ & -0.059 & $2.33 \%$ & $-42.72 \%$ \\
\hline ClassD_Basketba & -0.046 & $1.07 \%$ & $-40.43 \%$ & -0.066 & $1.78 \%$ & $-40.68 \%$ \\
\hline ClassD_BlowingBubbles & -0.059 & $1.35 \%$ & $-35.20 \%$ & -0.037 & $1.12 \%$ & $-35.25 \%$ \\
\hline ClassE_vidyo1 & -0.027 & $-0.32 \%$ & $-48.39 \%$ & -0.060 & $0.83 \%$ & $-53.40 \%$ \\
\hline ClassE_vidyo4 & -0.024 & $0.06 \%$ & $-47.58 \%$ & -0.056 & $1.07 \%$ & $-53.20 \%$ \\
\hline QP31 Averge & -0.034 & $0.69 \%$ & $-40.74 \%$ & -0.053 & $1.46 \%$ & $-44.52 \%$ \\
\hline ClassB_Cactus & -0.017 & $1.21 \%$ & $-39.16 \%$ & -0.054 & $1.75 \%$ & $-42.10 \%$ \\
\hline ClassB_ParkScene & -0.017 & & $-36.27 \%$ & -0.043 & $0.84 \%$ & $-41.96 \%$ \\
\hline ClassC_BasketballDrill & -0.062 & $0.98 \%$ & $-39.21 \%$ & -0.092 & $2.31 \%$ & $-44.17 \%$ \\
\hline ClassC_RaceHorses & -0.052 & $1.12 \%$ & $-42.82 \%$ & -0.063 & $2.42 \%$ & $-41.29 \%$ \\
\hline ClassD_BasketballPass & -0.081 & $0.87 \%$ & $-41.36 \%$ & -0.109 & $2.12 \%$ & $-39.43 \%$ \\
\hline ClassD_BlowingBubbles & -0.051 & $0.99 \%$ & $-35.73 \%$ & -0.044 & $1.41 \%$ & $-34.05 \%$ \\
\hline ClassE_vidyo1 & -0.015 & $0.30 \%$ & $-49.58 \%$ & -0.042 & $0.94 \%$ & $-53.60 \%$ \\
\hline ClassE_vidyo4 & -0.013 & $0.17 \%$ & $-48.52 \%$ & -0.051 & $1.18 \%$ & $-52.74 \%$ \\
\hline QP35 Averge & -0.039 & $0.77 \%$ & $-41.58 \%$ & -0.062 & $1.62 \%$ & $-43.67 \%$ \\
\hline
\end{tabular}

\section{REFERENCES}

[1] Advanced Video Coding, ITU-T Rec. H.264 and ISO/IEC 14496-10 (MPEG-4 AVC), Version 13, Mar. 2011.

[2] JCT-VC, "High Efficiency Video Coding (HEVC) Text Specification Draft 10,” JCTVC-L1003, 12th JCTVC meeting, Geneva, Jan. 2013.

[3] E. Peixoto and E. Izquierdo, "A Complexity-Scalable Transcoder from H.264/AVC to the New HEVC Codec," in Proc. IEEE International Conference on Image Processing (ICIP), Sep. 2012, pp. 737-740.

[4] X. Jing, W. C. Siu, L. P. Chau, and A. G. Constantinides, "Efficient Inter Mode Decision for H.263 to H.264 Video Transcoding Using Support Vector Machines," in Proc. IEEE International Symposium on Circuits and Systems (ISCAS), May 2009, pp. 2349-2352.

[5] D. Zhang, B. Li, J. Xu, and H. Li, "Fast Transcoding from H.264/AVC to High Efficiency Video Coding," in Proc. IEEE International Conference on Multimedia \& Expo. (ICME), July 2012, pp. 651-656. 
[6] R. Garrido-Cantos, J. D. Cock, J. L. Martínez, S. V. Leuven, and P. Cuenca, "Motion-Based Temporal Transcoding from H.264 AVC-to-SVC in Baseline Profile," IEEE Trans. Consum. Electron., vol. 57, no. 1, pp. 239-246, Feb. 2011.

[7] J. Zhang, F. Dai, Y. Zhang, and C. Yan, "Efficient HEVC to H.264/AVC Transcoding with Fast Intra Mode Decision," in Proc. the 19th International Conference on Multimedia Modeling, Jan. 2013, Lecture Notes in Computer Science vol. 7732, 2013, pp. 295-306.

[8] Z. Y. Chen, C. T. Tseng, and P. C. Chang, "Fast Inter Prediction for H.264 to HEVC Transcoding," in Proc. the 3rd International Conference on Multimedia Technology (ICMT), Guangzhou, China, Nov. 2013, Atlantis Press, pp. 1301-1308.

[9] Joint Video Team software JM17.2. Available at: https://iphome.hhi.de/suehring/tml/download/

[10] HM Reference Software 9.2. Available at: https://hevc.hhi.fraunhofer.de/svn/svn_HEVCSoft-ware 